\title{
Simulating AXAF Grating Spectra of Accreting White Dwarfs
}

\author{
Allyn F. Tennant ${ }^{1}$, Kinwah $\mathrm{Wu}^{2}$, Stephen L. O'Dell ${ }^{3}$ \\ and Martin C. Weisskopf ${ }^{4}$ \\ ${ }^{1}$ ES-84 Space Science Laboratory, NASA Marshall Space Flight Center, \\ Huntsville, AL 35812, USA \\ allyn.tennant@msfc.nasa.gov \\ ${ }^{2}$ Research Centre for Theoretical Astrophysics, School of Physics, \\ University of Sydney, NSW 2006, Australia \\ kinwah@physics.usyd.edu.au \\ ${ }^{3}$ ES-84 Space Science Laboratory, NASA Marshall Space Flight Center, \\ Huntsville, AL 35812, USA \\ odell@cosmos.msfc.nasa.gov \\ ${ }^{4}$ ES-01 Space Science Laboratory, NASA Marshall Space Flight Center, \\ Huntsville, AL 35812, USA \\ martin@smoker.msfc.nasa.gov
}

Received $199^{77}$ December 29, accepted 1998 August 1

\begin{abstract}
We present simulated AXAF spectra of accreting white dwarfs, using parameters appropriate for magnetic cataclysmic variables. The very high spectral resolution that can be obtained with the High-Energy Transmission Grating of AXAF can resolve the $\mathrm{keV} \mathrm{X}$-ray emission lines that characterise the temperature, density and velocity profiles of the shock-heated emission regions of these systems. These simulations demonstrate that actual spectra will allow us to place constraints on the white-dwarf mass and the accretion rate of the systems. The high-resolution spectra also allow the measurement of the velocity of the accretion flow in regions close to the white-dwarf surface.
\end{abstract}

Keywords: accretion — stars: binaries: close — stars: white dwarfs — data analysis

\section{Introduction}

The Advanced X-ray Astrophysics Facility (AXAF), to be launched in 1998, will provide revolutionary imaging and spectral capabilities in the energy range $0 \cdot 1-10 \mathrm{keV}$. (For an overview of the spacecraft and the instruments, see Weisskopf et al. 1995 and Weisskopf, O’Dell \& Van Speybroeck 1996.) At $E \sim 0.3 \mathrm{keV}$ the image quality will be such that more than 80 per cent of the photons detected from a point source will fall within $0 \cdot 5$-arcsec radius. The spatial resolution of AXAF is therefore comparable to that of ground-based optical telescopes. The transmission gratings on AXAF can achieve high spectral resolving powers. At low energies, the spectral resolving power $E / \Delta E$ exceeds 1000 (see AXAF Proposer's Guide, ASC.TD-402), which is adequate to resolve the $\mathrm{K} \alpha$ lines of the He- and $\mathrm{H}$-like ions of $\mathrm{O}(0.57$ and $0.65 \mathrm{keV}$ respectively), $\mathrm{Mg}(1.35$ and $1.47 \mathrm{keV}), \mathrm{Al}(1.60$ and $1.73 \mathrm{keV})$, Si $(1.86$ and $2 \cdot 00 \mathrm{keV}), \mathrm{S}(2 \cdot 46$ and $2 \cdot 62 \mathrm{keV})$, Ar $(3 \cdot 14$ and $3 \cdot 32 \mathrm{keV}), \mathrm{Ca}(3 \cdot 90$ and $4 \cdot 11 \mathrm{keV})$ and the $\mathrm{L}$ lines of $\mathrm{Fe}$ emitted from hot plasmas in various astrophysical environments, e.g. the dense, shock-heated accretion region near the surface of a white dwarf in a magnetic cataclysmic variable ( $\mathrm{mCV})$.

The currently best X-ray spectra of mCVs, obtained by the Solid-State Imaging Spectrometer (SIS) onboard the Advanced Satellite for Cosmology and Astrophysics (ASCA), show only the major emission lines above $1 \mathrm{keV}$ (see e.g. Fujimoto \& Ishida 1997). Theoretical calculations, however, show that many more lines are present (e.g. Cropper, Ramsay \& Wu 1998). These lines are useful not only for determining the white-dwarf mass, but also for diagnosing temperature and density structures of the accretion flow, and for deducing the relative chemical abundance of the accreting matter (Ishida 1991, 1997; Fujimoto \& Ishida 1997; Wu, Cropper \& Ramsay 1998).

We carry out simulation of AXAF grating spectra of accreting magnetic white dwarfs and investigate the diagnostic capability of the grating instruments. In the simulation, we first calculate the structure of the shock-heated emission regions and the raw 
Raw Spectra

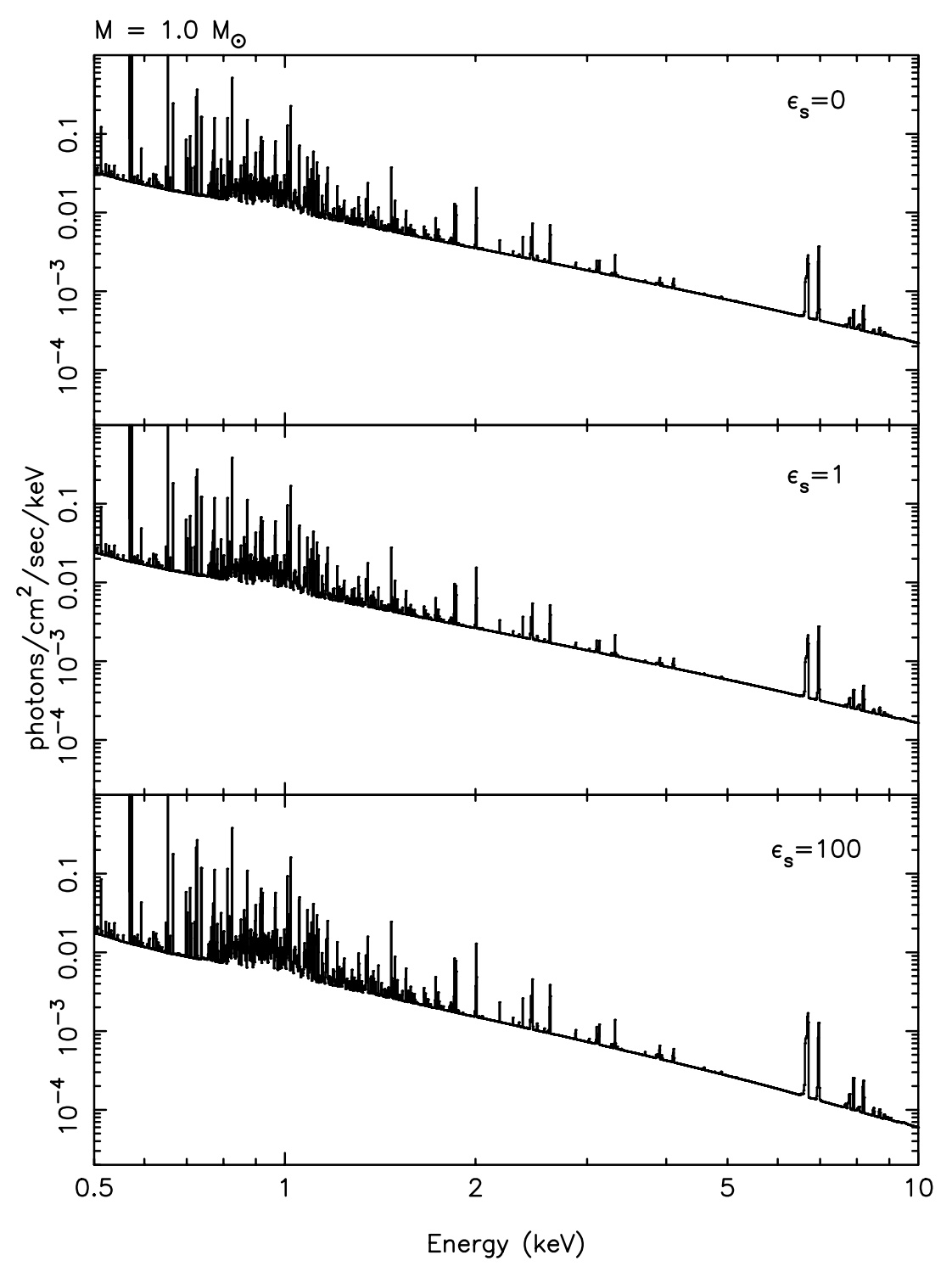

Figure 1-Raw spectra of $1 \cdot 0 M_{\odot}$ white dwarfs for $\epsilon_{s}=0,1$ and 100 .

spectra of its X-ray continuum and line emission. We then fold the raw spectra through a matrix that models the AXAF response. For this simulation we use the High-Energy Transmission Grating (HETG) (see Markert et al. 1994 for a detailed discussion of the HETG). The HETG comprises two grating arrays - the Medium-Energy Grating (MEG) and the High-Energy Grating (HEG). The gratings are mounted in the same structure, and so when the HETG is inserted two independent spectra, one from the MEG and the other from the HEG, ensue. The simulated AXAF response therefore includes the effects of the mirrors, the grating, and the AXAF CCD Imaging Spectrometer (ACIS) detector (Lumb et al. 1993). For a comparison of the spectral resolving capability of AXAF with those of previous instruments, we also carry out a simulation of spectra of the ASCA SIS (Tanaka, Inoue \& Holt 1994), which are CCD detectors without gratings.

\section{Simulation}

\section{1 Emission Region}

When supersonic matter is decelerated abruptly to settle onto the surface of an accreting white dwarf, a strong accretion shock is formed. The shock heats the accreting matter to a temperature $\sim 10 \mathrm{keV}$, causing it to ionise and to emit bremsstrahlung Xrays. Bremsstrahlung cooling is generally efficient as the shock-heated matter has very high temperatures $\left(\sim 10^{7}-10^{8} \mathrm{~K}\right)$ and densities $\left(\sim 10^{16} \mathrm{~cm}^{-3}\right)$. For white dwarfs with a strong ( $>1 \mathrm{MG}$ ) magnetic field, cyclotron cooling is also important, and for systems with low accretion rates and very strong $(\sim 10 \mathrm{MG})$ 


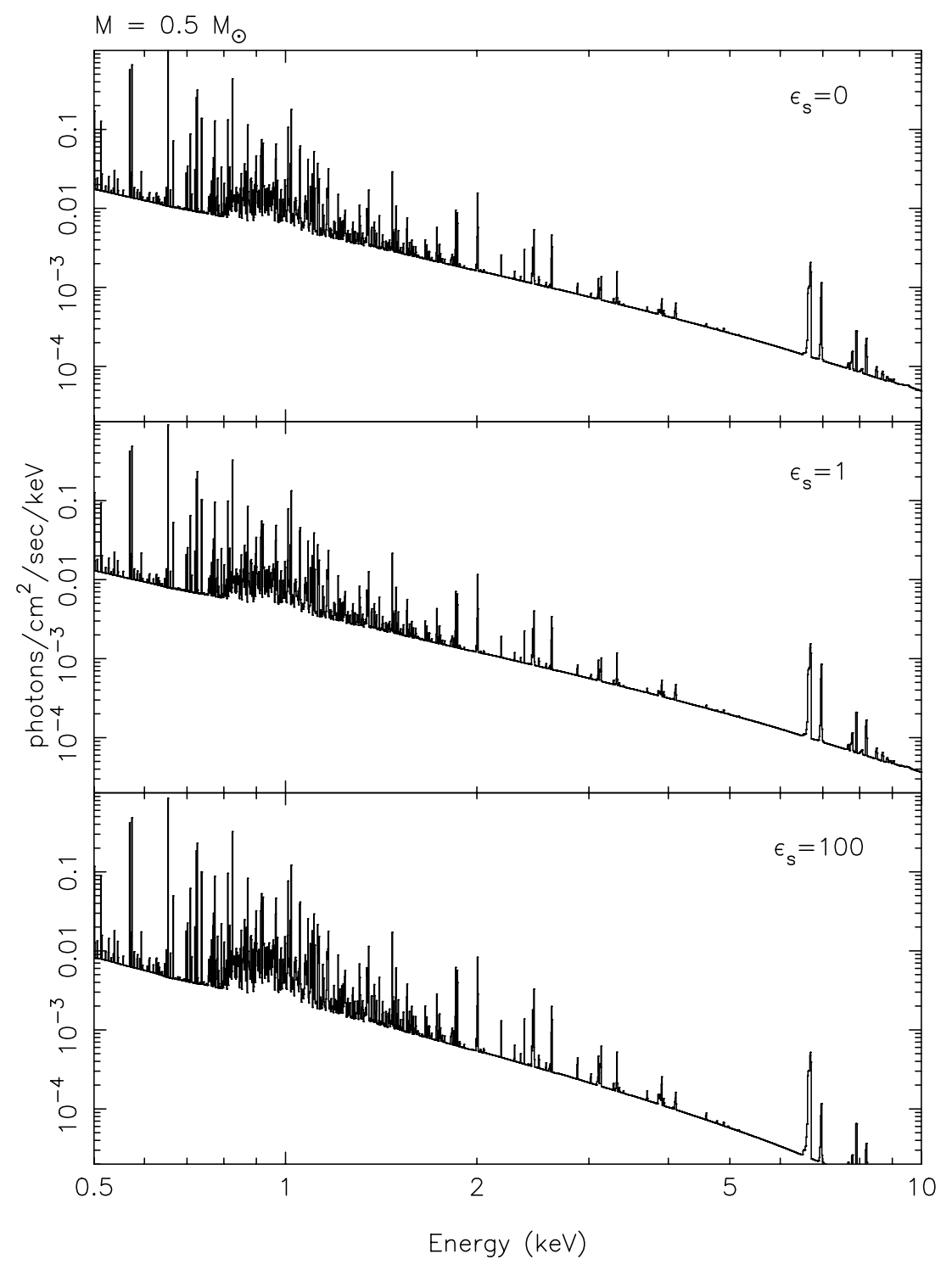

Figure 2-Same as Figure 1 but for $0 \cdot 5 M_{\odot}$ white dwarfs.

magnetic fields, it may be the more important process. The cooling processes determine the density and temperature structures of the shock-heated region and hence the properties of the radiation emitted from the region.

In this study we assume a simple one-dimensional planar model for the shock-heated emission regions of accreting magnetic white dwarfs. We use the hydrodynamic formulation given in $\mathrm{Wu}$, Chanmugam \& Shaviv (1994) (see also Wu 1994 and Cropper, Ramsay \& Wu 1998 for the correction) for accretion flow with a composite cooling process to determine the density and temperature structures of the emission region. We assume that only bremsstrahlung and cyclotron cooling are important, and ignore other cooling processes. We divide the shock-heated emission region into 200 vertically stratified zones.
The sizes of the zones are chosen such that the difference between the flow velocities at the zone boundaries is $1 / 200$ of the pre-shock free-fall velocity in order to avoid unphysical weighing of the emission from the dense bottom zones near the white dwarf surface. The local effective temperature and density are calculated from the mean flow velocity of the zones.

We assume the coronal condition (Wilson 1962; Mewe 1990), so that the line and continuum radiation emitted from each zone can be calculated using the MEKAL opacity code (Mewe, Gronenschild \& van den Oord 1985; Mewe, Kaastra \& Liedahl 1995) from XSPEC (Arnaud 1996). The total emission is the sum of the contributions from all zones in the shock-heated region.

To avoid unnecessary complication in this demonstration study, we ignore effects due to absorption 


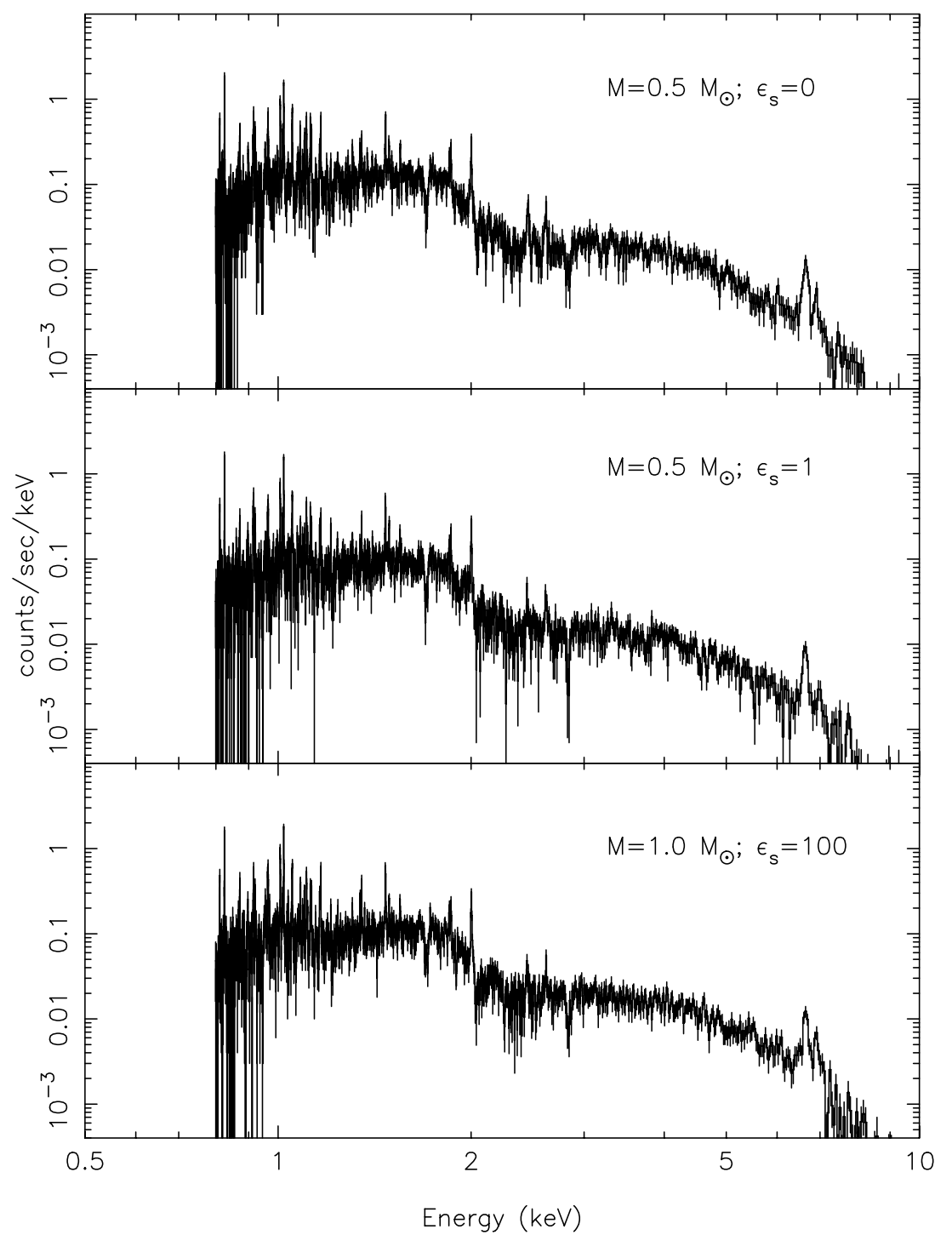

Figure 3-Simulated HEG spectra of accreting white dwarfs with $\left(M_{w d} / M_{\odot}, \epsilon_{s}\right)=(0 \cdot 5,0)$, $(0 \cdot 5,1)$ and $(1 \cdot 0,100)$. Most of the variations in the continuum are due to variations in the instrument sensitivity. The grating disperses the photons in both the plus and minus directions over several CCD chips. For these simulations, we assume the two spectra have been added. However, the gaps between the chips cause the drops near $0 \cdot 95,1 \cdot 2,1 \cdot 7,2 \cdot 8$ and $8.5 \mathrm{keV}$. The mirrors are coated with Ir which has strong absorption edges just above $2 \mathrm{keV}$.

by the cool material surrounding the shock-heated region (Done, Osborne \& Beardmore 1995; Mukai, Ishida \& Osborne 1997) and effects due to reflection from the white-dwarf surface (van Teeseling, Kaastra $\&$ Heise 1996). These effects, which are strongly source dependent, may be important and should be considered in analysing the actual data (e.g. Cropper, Ramsay \& Wu 1998).

We consider cases for white dwarfs with masses $M_{w d}$ of $0 \cdot 5 M_{\odot}$ and $1 \cdot 0 M_{\odot}$. We use the Nauenberg (1972) mass-radius relation to determine the white dwarf radius. The relative strengths of the bremsstrahlung and cyclotron cooling processes are determined by the white-dwarf magnetic field and the accretion rate; and they are specified by a parameter $\epsilon_{s}$, which is the ratio of the bremsstrahlungcooling timescale to the cyclotron-cooling timescale at the accretion-shock surface. For $\epsilon_{s}=0$, only bremsstrahlung cooling is present; for $\epsilon_{s}=1$, both bremsstrahlung and cyclotron cooling have equal strength at the shock surface; for $\epsilon_{s}=100$, cyclotron cooling is the dominant process at the shock surface. We fix the specific accretion rate to be $1 \mathrm{~g} \mathrm{~cm}^{-2} \mathrm{~s}^{-1}$; large $\epsilon_{s}$ corresponds to a strong white-dwarf magnetic 


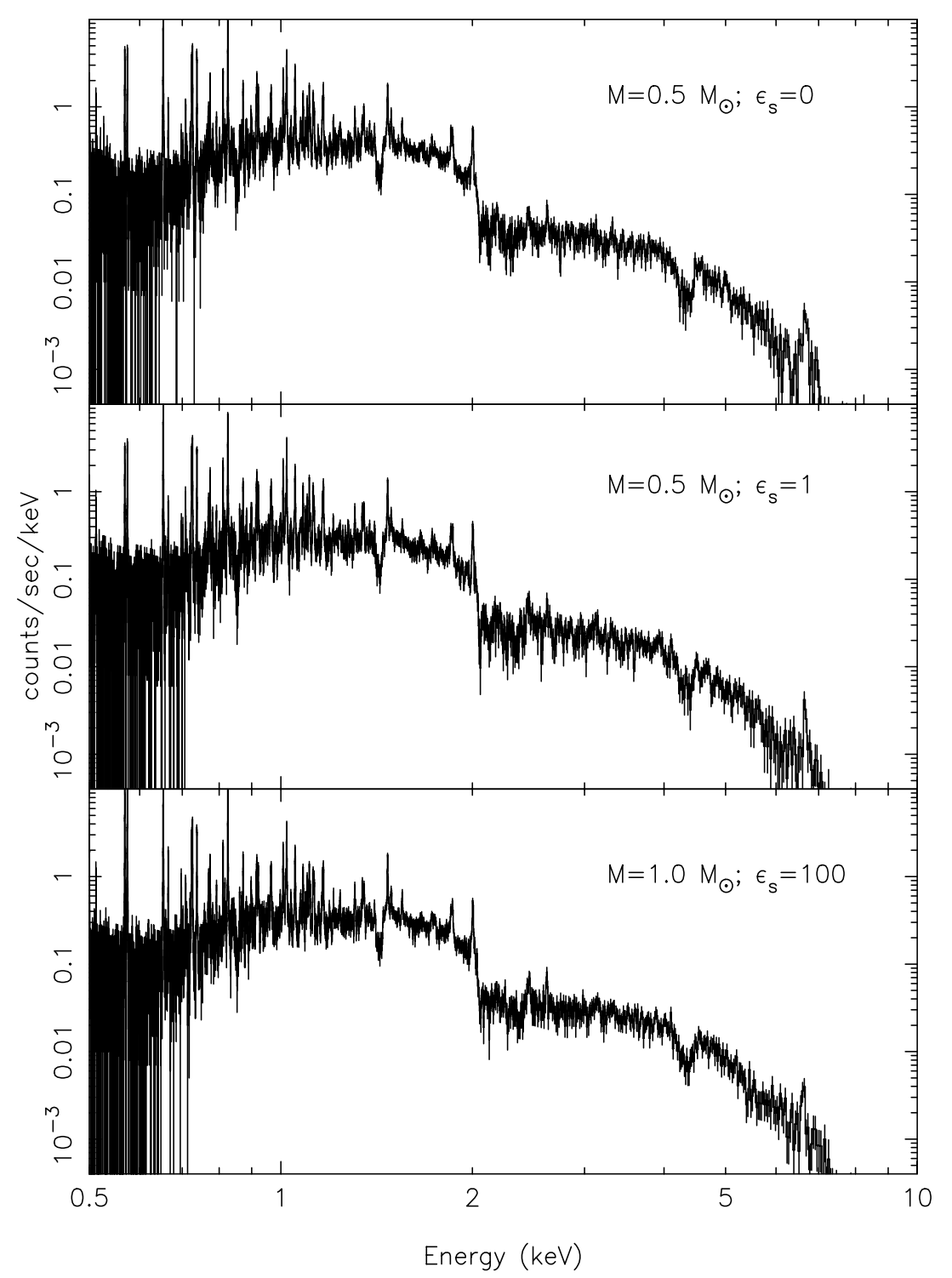

Figure 4-Same as Figure 3 but for the MEG. Here the chip boundaries occur near $0 \cdot 85,1 \cdot 4$ and $4 \cdot 2 \mathrm{keV}$.

field. (If we fix the magnetic field instead, then larger $\epsilon_{s}$ corresponds to lower accretion rates.- - see Wu, Chanmugam \& Shaviv 1994 for the dependence of $\epsilon_{s}$ on the white-dwarf magnetic field and the accretion rate.) We set the cross-section area of the emission region to be $2.5 \times 10^{14} \mathrm{~cm}^{2}$ and the distance of the system to be $100 \mathrm{pc}$, and assume solar abundances.

\subsection{Spectral Synthesis}

We calculate the raw input spectra, using the temperature and density structures determined by the hydrodynamic calculations and the MEKAL code for the X-ray emission. Figure 1 shows the raw spectra of the systems with a $1 \cdot 0 M_{\odot}$ white dwarf and $\epsilon_{s}=0,1$ and 100 . As the resolution of the spectra is limited mainly by the binning process, the emission lines all appear to be sharp and precisely resolved. The $\mathrm{K} \alpha$ lines of $\mathrm{H}$ - and He-like ions of $\mathrm{O}, \mathrm{Mg}, \mathrm{Si}, \mathrm{S}, \mathrm{Ar}, \mathrm{Ca}$ and the $\mathrm{Fe}$ lines are clearly seen. The Fe L lines are prominent, with hints that the strength of the lines increase with $\epsilon_{s}$, the efficiency of cyclotron cooling relative to bremsstrahlung cooling. In Figure 2 the raw spectra of the $0 \cdot 5 M_{\odot}$ accreting white dwarfs are shown. The general features in the spectra of the $0 \cdot 5 M_{\odot}$ white dwarfs are similar to those in the spectra of the $1 \cdot 0 M_{\odot}$ white dwarfs, except that the emission lines below $2 \mathrm{keV}$ are generally greater and the continua are weaker. Moreover, the strength of the Fe $\mathrm{L}$ lines are also stronger for $\epsilon_{s}=100$ than for $\epsilon_{s}=0$ and 1 . 


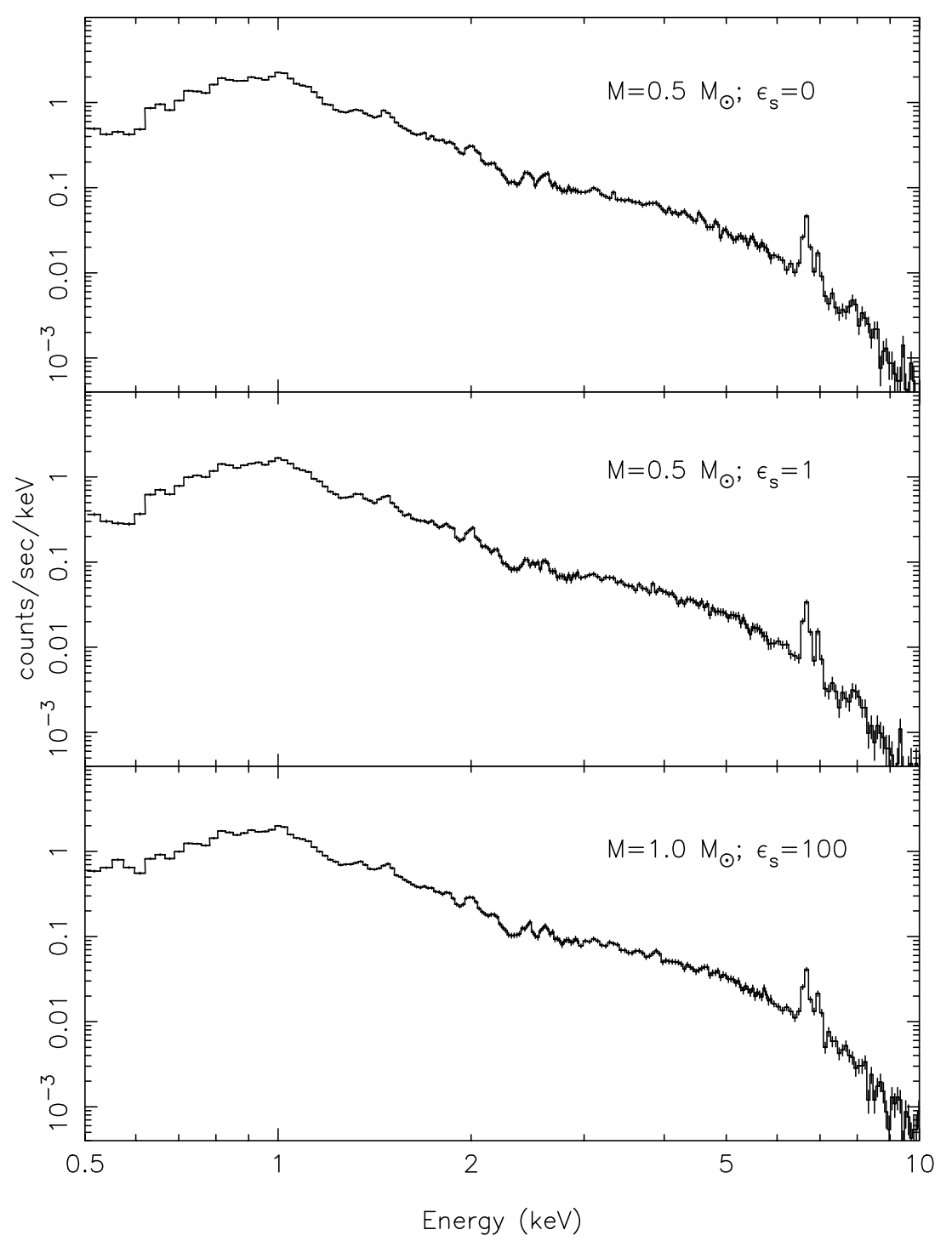

Figure 5-Simulated ASCA SIS spectra of accreting white dwarfs with parameters the same as those in Figures 3 and 4.

The system parameters we have assumed in our simulation are similar to the system AM Herculis itself, whose white-dwarf mass is about $1 M_{\odot}$ (Cropper, Ramsay \& Wu 1998), magnetic field is about 20 MG (Chanmugam 1992), and distance is about $75 \mathrm{pc}$ (Cropper 1990). To verify the normalisation in our simulation we consider the case for the $1 \cdot 0 M_{\odot}$ white dwarf and fold the raw spectra through the EXOSAT ME response. We obtain the count rates $4 \cdot 1,3 \cdot 1$ and $1 \cdot 6 \mathrm{ct} / \mathrm{s}$ at $1-10 \mathrm{keV}$ for $\epsilon_{s}=0,1$ and 100 respectively. These count rates are consistent with the rate of $4.5 \mathrm{ct} / \mathrm{s}$, derived from the EXOSAT ME observation of the system AM Herculis itself in Aug 10, 1983 (data were obtained from the High Energy Astrophysics Science Archive Research Center).
In Figure 3 we show the simulated HEG spectra of white dwarfs with parameters $\left(M_{w d} / M_{\odot}, \epsilon_{s}\right)=(0 \cdot 5$, $0),(0 \cdot 5,1)$ and $(1 \cdot 0,100)$ for an integration time of $100 \mathrm{ksec}$. The photon counts and the corresponding Poisson noise of the spectra are simulated. The K $\alpha$ lines of $\mathrm{Mg}, \mathrm{Si}, \mathrm{S}, \mathrm{Ar}, \mathrm{Ca}$ and $\mathrm{Fe}$ are well resolved in the spectra. In Figure 4 the MEG spectra for the same 'observing' conditions are shown. The MEG, which offers better effective area below about $4 \mathrm{keV}$, clearly resolves the $\mathrm{O}, \mathrm{Mg}$ and the $\mathrm{Fe} \mathrm{L}$ lines.

For comparison, we also simulate the X-ray spectra for an instrument without gratings. We use the ASCA SIS as a demonstration. We fold the raw spectra through the ASCA SIS response function, for an integration time of $100 \mathrm{ksec}$. The $\mathrm{K} \alpha$ lines of $\mathrm{Si}, \mathrm{S}$ and $\mathrm{Ar}$ are still visible (Figure 5), but are not 


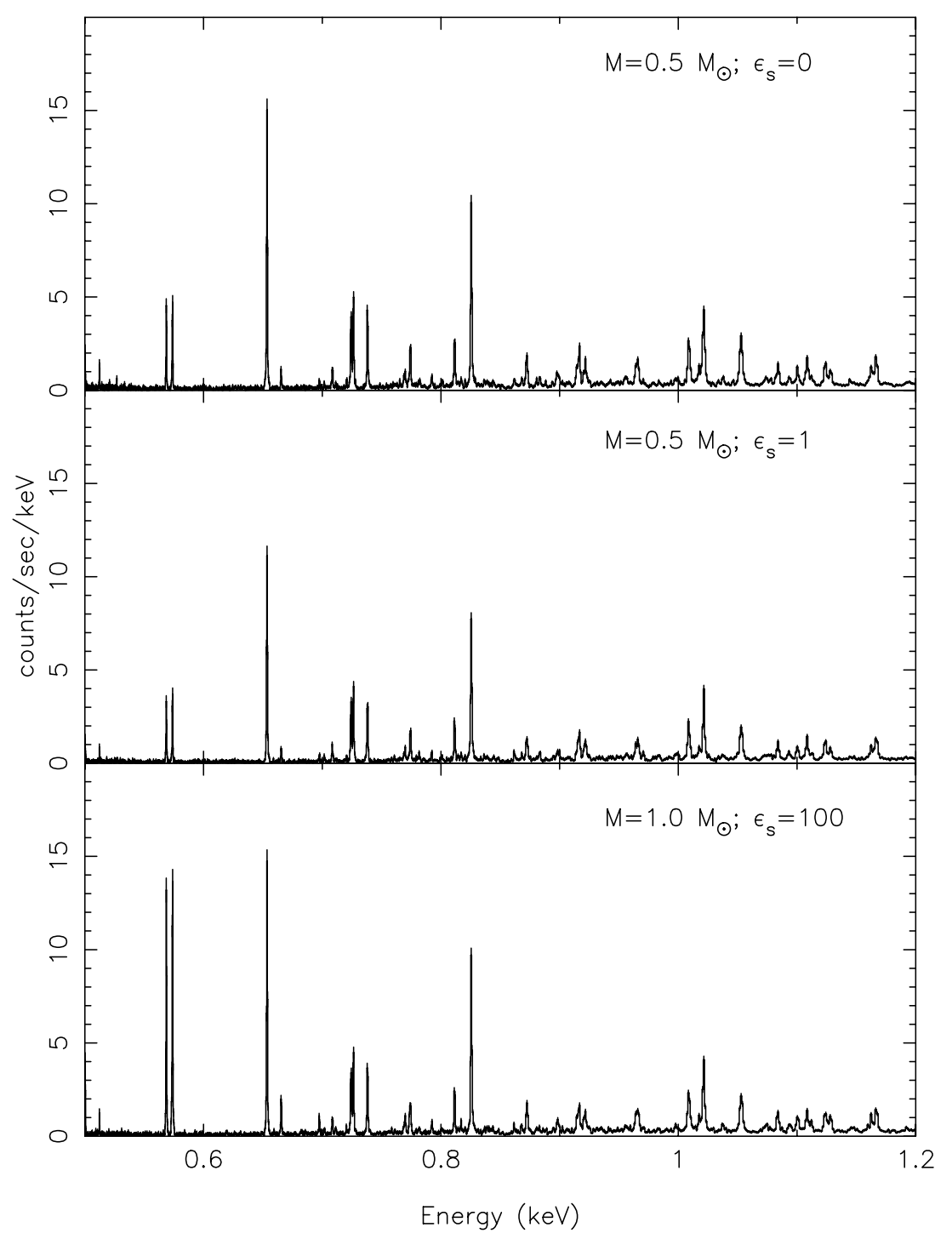

Figure 6-Simulated MEG spectra in linear scales of photon count and energy. The parameters of the simulations are the same as those in Figures 3 and 4.

as obvious as those in the AXAF grating spectra. The Mg lines now blend with the lower excitation $\mathrm{Fe}$ lines; the $\mathrm{Ca}$ and $\mathrm{O}$ lines are no longer clearly visible, and the Fe L lines are smeared beyond the resolution of the instrument.

\section{Diagnostic Capabilities}

Because of temperature and density stratification in the emission region, emission lines of different elements are emitted from different heights above the white dwarf surface. The free-fall velocity at the surface of a $0 \cdot 5 M_{\odot}$ white dwarf is $\sim 3 \times 10^{8} \mathrm{~cm} \mathrm{~s}^{-1}$. This will cause an energy shift of about 1 part in 100 for the centre energies of the emission lines. For typical $\mathrm{mCV}$ parameters (e.g. a specific accretion rate of about $1 \mathrm{~g} \mathrm{~cm}^{2} \mathrm{~s}^{-1}$ ), the emission from the shock-heated region is optically thick in the optical bands. The capability of optical spectroscopy is therefore limited, despite the fact it can provide a very high velocity resolution.

The $\mathrm{keV}$ emission lines, however, can be optically thin down to about $10 \mathrm{~m}$ above the white dwarf surface. The spectral resolving power $(E / \Delta E)$ of 1000 provided by the AXAF gratings implies a velocity resolution of $\sim 3 \times 10^{7} \mathrm{~cm} \mathrm{~s}^{-1}$, which is sufficient to resolve the velocity shift of the lines from the post-shock matter of an accreting white dwarf.

We show in Figure 6 the simulated MEG spectra over the energy range $0.5-1.2 \mathrm{keV}$ with linear scales in photon counts and energy for accreting white dwarfs with the same parameters as those in Figures 3 and 4 . This demonstrates that in spite of the noise in the spectra shown in Figure 4, lines such 


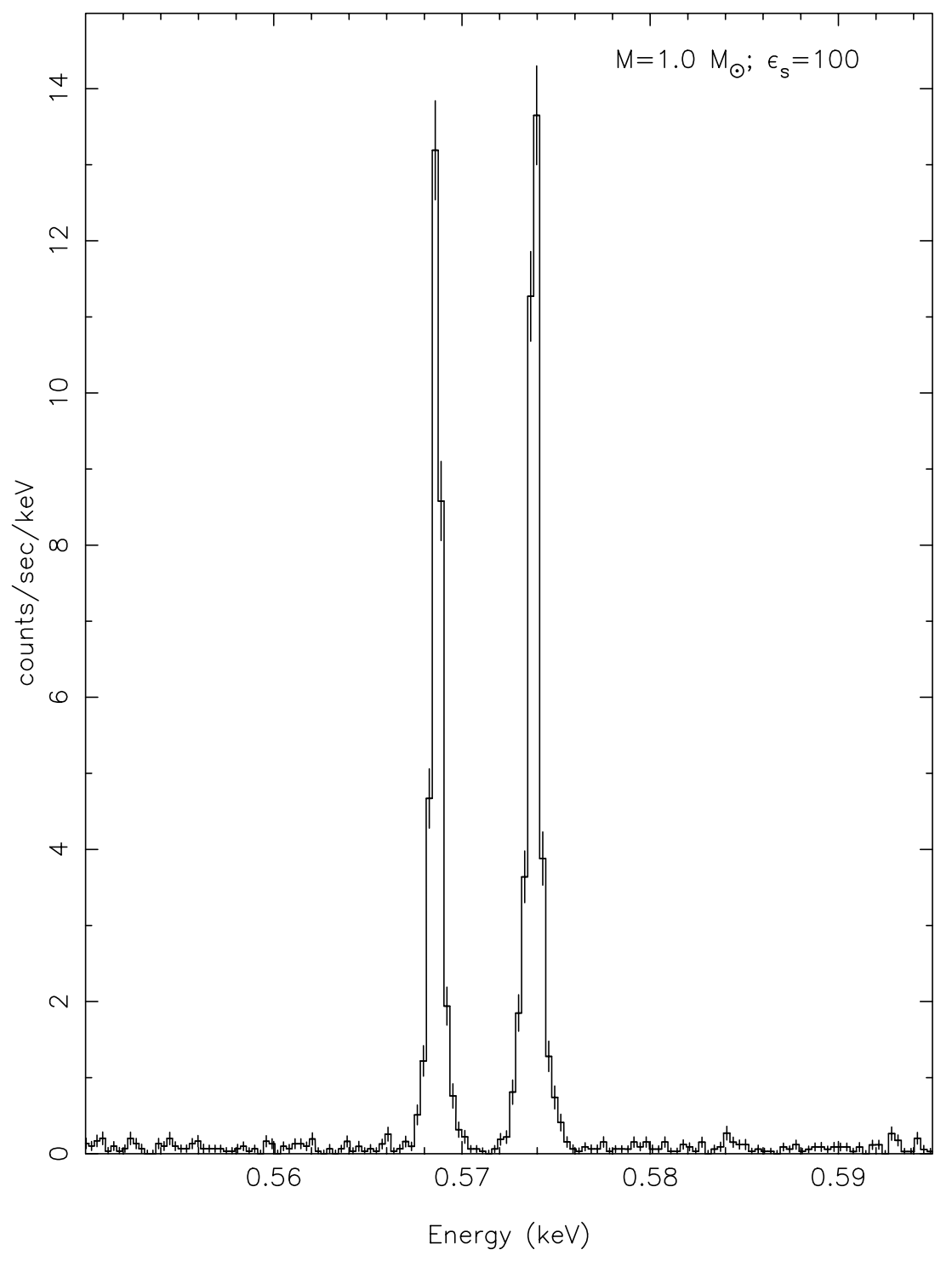

Figure 7-Simulated O VII He4 and He5 lines for $M_{w d}=1 \cdot 0 M_{\odot}$ and $\epsilon_{s}=100$.

as the O VII Lyman- $\alpha$ line (at $0.653 \mathrm{keV}$ ), and the $\mathrm{O}$ VII He4 and He5 lines (at $0.574 \mathrm{keV}$ and $0 \cdot 569$ $\mathrm{keV}$ respectively) are actually strongly detected and well resolved by the MEG. The forbidden O VII He6 line (at $0.561 \mathrm{keV}$ ) is, however, undetected because it is suppressed (see e.g. Mewe 1990) by the high density $\left(\gg 10^{-12} \mathrm{~g} \mathrm{~cm}^{-3}\right)$ in the emission region. Since the line features in the three cases we consider are distinguishable, the grating spectra to be obtained by AXAF can be used to constrain the system parameters such as the white dwarf mass and the parameter $\epsilon_{s}$. As $\epsilon_{s}$ depends on the accretion rate, the magnetic field and the mass of the white dwarf, if the mass and the magnetic field are known, one may also deduce the accretion rate.

In Figure 7 we show the simulation of the $\mathrm{O}$ VII He4 and He5 lines emitted from a system with
$M_{w d}=1 \cdot 0 M_{\odot}$ and $\epsilon_{s}=100$. Although the two lines appear to have about the same amplitude, their fluxes are actually quite different. There are about 1000 counts in the He5 line and 1300 counts in the He4 line. The same apparent amplitude for the lines is due to the binning effect, i.e. the centre of the He5 line is closer to the centre of a bin, whereas the centre of the $\mathrm{He} 4$ line is closer the boundary between two bins.

We model the lines with Lorentzians and the continuum with a constant term and obtain the $1 \sigma$ uncertainty in the location of the line centres of about $0 \cdot 01 \mathrm{eV}$. The uncertainty in the mean is generally equal to the uncertainty in the population divided by the square root of the number of measurements. When the line dominates the continuum, each photon detection in the line is an independent 
measurement of the line centre, i.e. each photon independently has a $68 \%$ chance of falling within one (instrumental) $\sigma$ of the line centre. Therefore, we have $\sigma_{c}=\sigma_{i} / N^{\frac{1}{2}}$, where $\sigma_{i}$ is the instrumental width, $\sigma_{c}$ the uncertainty in the line centre energy, and $N$ the number of photons in the line. At the energy range $\sim 0.57 \mathrm{keV}, \sigma_{i}$ is about $0.3 \mathrm{eV}$. Hence, for 1000 photons the uncertainty in the line centre is $0.01 \mathrm{eV}$, consistent with what we have measured from the simulation.

We expect the systematic error of ACIS to be much less than $10 \%$. If we increase the uncertainty in the flux for every point by $10 \%$ to allow for systematic errors, we find that the formal $1 \sigma$ uncertainty in the line position increases only to $0.04 \mathrm{eV}$. This value is still better than the instrumental resolution. Therefore, provided that there are enough counts to detect the line, say 10 or more, one should be able to locate the centre accurately.

The instrumental resolution at the O VII lines implies that the velocity of the accreting matter which emits the lines can be measured to an accuracy better than $1.6 \times 10^{7} \mathrm{~cm} \mathrm{~s}^{-1}$. With 1000 counts in the line and no systematic errors (i.e. an uncertainty of $0 \cdot 01 \mathrm{eV}$ ), the velocity uncertainty is $5 \times 10^{5} \mathrm{cms}^{-1}$. Thus, if we divide the data into 10 orbital phase bins (for the measurement of the variations in the velocity due to orbital motion) and allow the line flux to be weakened by a factor of a few, a velocity resolution of $\sim 10^{7} \mathrm{~cm} \mathrm{~s}^{-1}$ can still be achieved. As we have presented a very conservative estimate, higher velocity resolution is achievable if strong lines are used and/or the sums of several lines are considered. Our simulations therefore have shown that the AXAF gratings will be able to diagnose accretion flow in regions as close as $10 \mathrm{~m}$ above the white dwarf surface.

In summary, we have simulated AXAF grating spectra of accreting white dwarfs, using parameters appropriate for magnetic cataclysmic variables. Our simulations show that the High-Energy Transmission Grating of AXAF can resolve the keV X-ray emission lines that characterise the temperature, density and velocity profiles of the shock-heated emission regions of these systems. This will allow us to place constraints on the white-dwarf mass and the accretion rate. The high-resolution spectra will also enable us to directly measure the velocity of accretion flow in regions very close to the white-dwarf surface.

\section{Acknowledgments}

We thank K. Arnaud for providing the MEG and HEG response matrices for our preliminary calculations and M. Cropper for discussions and for pointing out the errors in our previous calculations. $\mathrm{KW}$ acknowledges the support from ARC through an Australian Research Fellowship. This research has made use of data obtained through the High Energy Astrophysics Science Archive Research Center Online Service, provided by the NASA Goddard Space Flight Center.

\section{References}

Arnaud, K. A. 1996, in Astronomical Data Analysis Software and Systems V, eds G. Jacoby \& J. Barnes, ASP Conf. Series 101 (San Francisco: ASP), p. 17

Chanmugam, G. 1992, ARA\&A, 30, 143

Cropper, M. 1990, Space Sci. Rev., 54, 195

Cropper, M., Ramsay, G., \& Wu, K. 1998, MNRAS, 293, 222

Done, C., Osborne, J. P., \& Beardmore, A. P. 1995, MNRAS, 276,483

Fujimoto, R., \& Ishida, M. 1997, ApJ, 474, 774

Ishida, M. 1991, PhD Thesis, University of Tokyo

Ishida, M. 1997, in X-ray Imaging and Spectroscopy of Cosmic Hot Plasmas, eds F. Makino \& K. Mitsuda (Tokyo: Universal Academy Press), p. 537

Lumb, D. H., et al. 1993, Proc. SPIE, 2006, 265

Markert, T. H., et al. 1994, in EUV, X-ray and Gamma-ray Instrumentation for Astronomy V, eds O. H. W. Siegmund \& J. V. Vallerga, Proc. SPIE, 2280, 168

Mewe, R. 1990, in Physical Process in Hot Cosmic Plasmas, NATO ASI Series, eds W. Brinkmann et al. (Dordrecht: Kluwer), p. 39

Mewe, R., Gronenschild, E. H. B. M., \& van den Oord, G. H. J. 1985, A\&AS, 62, 197

Mewe, R., Kaastra, D. S., \& Liedahl, D. A. 1995, Legacy (Journal of HEASARC), 6, 16

Mukai, K., Ishida, M., \& Osborne, J. 1997, in X-ray Imaging and Spectroscopy of Cosmic Hot Plasmas, eds F. Makino \& K. Mitsuda (Tokyo: Universal Academy Press), p. 543 Nauenberg, M. 1972, ApJ, 175, 417

Tanaka, Y., Inoue, H., \& Holt, S. S. 1994, PASJ, 46, L47 van Teeseling, A., Kaastra, J. S., \& Heise, J. 1996, A\&A, 312,186

Weisskopf, M. C., O'Dell, S. L., Elsner, R. F., \& Van Speybroeck, L. P. 1995, Proc. SPIE, 2515, 312

Weisskopf, M. C., O’Dell, S. L., \& Van Speybroeck, L. P. 1996, Proc. SPIE, 2805, 2

Wilson, R. 1962, Quant. Spectr. Rad. Transf., 2, 477

Wu, K. 1994, PASA, 11, 61

Wu, K., Chanmugam, G., \& Shaviv, G. 1994, ApJ, 426, 664

Wu, K., Cropper, M., \& Ramsay, G. 1998, in preparation 